\title{
Targeting cysteine-mediated dimerization of the MUC1-C oncoprotein in human cancer cells
}

\author{
DEEPAK RAINA ${ }^{2}$, REHAN AHMAD ${ }^{1}$, HASAN RAJABI $^{1}$, GOVIND PANCHAMOORTHY $^{2}$, \\ SURENDER KHARBANDA ${ }^{2}$ and DONALD KUFE ${ }^{1}$ \\ ${ }^{1}$ Dana-Farber Cancer Institute, Harvard Medical School, and ${ }^{2}$ Genus Oncology, Boston, MA, USA
}

Received November 4, 2011; Accepted December 9, 2011

DOI: $10.3892 /$ ijo.2011.1308

\begin{abstract}
The MUC1 heterodimeric protein is aberrantly overexpressed in diverse human carcinomas and contributes to the malignant phenotype. The MUC1-C transmembrane subunit contains a CQC motif in the cytoplasmic domain that has been implicated in the formation of dimers and in its oncogenic function. The present study demonstrates that MUC1-C forms dimers in human breast and lung cancer cells. MUC1-C dimerization was detectable in the cytoplasm and was independent of MUC1-N, the N-terminal mucin subunit that extends outside the cell. We show that the MUC1-C cytoplasmic domain forms dimers in vitro that are disrupted by reducing agents. Moreover, dimerization of the MUC1-C subunit in cancer cells was blocked by reducing agents and increased by oxidative stress, supporting involvement of the CQC motif in forming disulfide bonds. In support of these observations, mutation of the MUC1-C CQC motif to AQA completely blocked MUC1-C dimerization. Importantly, this study was performed with MUC1-C devoid of fluorescent proteins, such as GFP, CFP and YFP. In this regard, we show that GFP, CFP and YFP themselves form dimers that are readily detectable with cross-linking agents. The present results further demonstrate that a cell-penetrating peptide that targets the MUC1-C CQC cysteines blocks MUC1-C dimerization in cancer cells. These findings provide definitive evidence that: i) the MUC1-C cytoplasmic domain cysteines are necessary and sufficient for MUC1-C dimerization, and ii) these CQC motif cysteines represent an Achilles' heel for targeting MUC1-C function.
\end{abstract}

Correspondence to: Dr Donald Kufe, Department of Medical Oncology, Dana-Farber Cancer Institute, 450 Brookline Avenue, Dana 830, Boston, MA 02215-5450, USA

E-mail: donald_kufe@dfci.harvard.edu

Abbreviations: MUC1, mucin 1; MUC1-C, MUC1 C-terminal subunit; MUC1-CD, MUC1 cytoplasmic domain; DTT, dithiothreitol; GFP, green fluorescence protein; DSS, disuccinimidyl suberate; BME, $\beta$-mercaptoethanol

Key words: MUC1-C, dimerization, reactive cysteines, oxidation

\section{Introduction}

Mucins are characterized by the presence of tandem repeat structures that are extensively glycosylated through GalNAc $O$-linkages at threonine and serine residues (1). The human mucin (MUC1 to MUC21) family consists of secreted and transmembrane forms that contribute to a physical barrier, which protects the apical borders of epithelial cells from the external environment. The transmembrane mucin 1 (MUC1) has a sea urchin sperm protein-enterokinase-agrin (SEA) domain that undergoes autocleavage, resulting in two subunits that in turn form a stable heterodimer at the cell membrane (1). The MUC1 $\mathrm{N}$-terminal (MUC1-N) subunit contains the glycosylated tandem repeats and extends beyond the glycocalyx of the cell as part of the protective physical barrier. The MUC1 C-terminal (MUC1-C) subunit consists of a 58-amino acid (aa) extracellular domain, a 28-aa transmembrane domain and a 72-aa cytoplasmic domain (1). The MUC1-N/MUC1-C heterodimer localizes to the apical border of normal secretory epithelial cells. However, upon loss of polarity associated with transformation, the MUC1 heterodimeric complex is expressed at increased levels and over the entire surface of carcinoma cells (2). With loss of restriction to the apical membrane, the MUC1 heterodimer forms complexes with the epidermal growth factor receptor (EGFR) and other members of the ErbB family $(3,4)$. The overexpression of MUC1 by carcinoma cells is also associated with localization of MUC1-C to the cytoplasm and targeting of this subunit to the nucleus and mitochondria (1). Significantly, overexpression of MUC1-C and, specifically, the MUC1-C cytoplasmic domain is sufficient to induce anchorage-independent growth and tumorigenicity $(5,6)$.

MUC1-C interacts with diverse effectors that have been linked to transformation (1). The MUC1-C cytoplasmic domain is phosphorylated by EGFR, MET, SRC, ABL, protein kinase C and glycogen synthase kinase $3 \beta$ (1). In addition, the MUC1-C cytoplasmic domain binds directly to the Wnt effector $\beta$-catenin (6,7), p53 (8), NF-кB RelA (9), and STAT3 (10). In this respect, MUC1-C associates with diverse transcription factors on the promoters of their target genes and contributes to the regulation of gene expression (11). Nuclear localization of MUC1-C is dependent on the formation of homodimers and is mediated by importin $\beta$ and the nucleoporin Nup62 (12). The MUC1-C cytoplasmic domain contains a CQC motif that has been associated with the formation of homodimers (12). In support of this model, 
mutation of the CQC motif blocks dimerization and localization of MUC1-C to the nucleus (12). In addition, mutation of the MUC1-C CQC motif blocks MUC1-C-induced transcriptional coactivation, anchorage-independent growth and tumorigenicity (12). These results indicated that targeting of the MUC1-C CQC motif could block the MUC1-C transforming function. Indeed, cell-pentrating peptide inhibitors that bind to the MUC1-C cytoplasmic domain at the CQC motif have been effective in inducing death of breast, prostate, lung and other types of cancer cells growing in vitro and as xenografts in immunocompromised mice (13-16). Thus, a detailed understanding of how the MUC1-C subunit undergoes dimerization is of importance to the targeting of this oncoprotein.

The present study has characterized the formation of MUC1-C dimers in human cancer cells. The results demonstrate that MUC1-C constitutively forms dimers and that these complexes are detectable in the cytoplasm. The results also demonstrate that MUC1-C dimer formation is strictly dependent on the CQC motif and that targeting these cysteine residues is sufficient to block function of the MUC1-C cytoplasmic domain in conferring these covalent complexes.

\section{Materials and methods}

Cell culture. Human ZR-75-1, H1975 and LNCaP/MUC1-C cells were grown in RPMI-1640 medium supplemented with $10 \%$ heat-inactivated fetal bovine serum (HI-FBS), $100 \mathrm{U} / \mathrm{ml}$ penicillin, $100 \mu \mathrm{g} / \mathrm{ml}$ streptomycin and $2 \mathrm{mM}$ L-glutamine. Human MCF-7 breast cancer cells, HCT116/MUC1, HCT116/MUC1-C, HCT116/MUC1(AQA) and 293 cells were grown in DMEM with $10 \%$ HI-FBS, antibiotics, and $2 \mathrm{mM}$ L-glutamine. Cells were treated with GO-203 (AnaSpec) and hydrogen peroxide (Sigma).

Immunoblot analysis. Total cell lysates were prepared as described with the exclusion of dithiothreitol (DTT) from the lysis buffer (13). Cytoplasmic and particulate lysates were prepared in the absence of DTT using the cell fractionation kit (BioVision). Soluble proteins were separated in non-reducing SDS-polyacrylamide gels and immunoblotted with anti-MUC1C (17), anti- $\beta$-actin (Sigma) and anti-GFP (Cell Signaling Technology). Reactivity was detected with horseradish peroxidase-conjugated secondary antibodies and chemiluminescence.

In vitro binding assays. Purified His-MUC1-CD was incubated with or without DTT in the binding buffer (PBS/1\% NP-40) for $1 \mathrm{~h}$ at room temperature. The proteins were separated in a non-reducing SDS-polyacrylamide gel and analyzed by immunoblotting.

Cell transfection and protein cross-linking. Cells (293) were transfected with pHR-CMV-GFP, pcDNA-CFP and pcDNAYFP plasmids (Addgene) in the presence of Lipofectamine. After $48 \mathrm{~h}$, cell pellets were suspended in $500 \mu \mathrm{l}$ of PBS with the addition of freshly prepared disuccinimidyl suberate (DSS; Thermo Scientific) to a final concentration of $5 \mathrm{mM}$ and incubated by rotation at room temperature for $30 \mathrm{~min}$. The cells were then pelletted, resuspended in RIPA buffer, incubated on ice for $10 \mathrm{~min}$ and centrifuged at $14,000 \mathrm{rpm}$ for $10 \mathrm{~min}$. Supernatants were collected and mixed with Laemmli sample buffer for analysis by SDS-PAGE and immunoblotting.

\section{Results}

MUC1-C subunitforms dimers in carcinomacells. The MUC1-C subunit consists of a 58-aa extracellular domain that is glycosylated on Asn-36 and a 28-aa transmembrane region (Fig. 1A). The MUC1-C 72-aa cytoplasmic domain includes a CQC motif (aa 87-89) that has been implicated in the formation of dimers (12) (Fig. 1A). In this regard, the CQC motif is adjacent to RRK, thus potentially increasing reactivity of the thiols by the neighboring positively charged side chains $(18,19)$ (Fig. 1A). With the exception of a single Cys residue in the transmembrane region, there are no other cysteines in MUC1-C. Immunoblot analysis of human ZR-75-1 breast cancer cells for MUC1-C expression demonstrated a $17-\mathrm{kDa}$ species that is the unglycosylated form (3) (Fig. 1B). The broader $25-20 \mathrm{kDa}$ immunoreactive species is the MUC1-C form that is glycosylated on Asn-36 (3) (Fig. 1B). In addition, immunoreactivity was detected at $\sim 50 \mathrm{kDa}$, consistent with the formation of homodimers (Fig. 1B). Immunoblot analysis of lysates from human MCF-7 breast cancer and H1975 lung cancer cells demonstrated the same pattern of detection with monomers and dimers (Fig. 1C). These findings provided support for the formation of MUC1-C homodimers in breast and lung cancer cells.

Dimerization of MUC1-C is independent of the MUC1-N subunit. MUC1-C forms a heterodimeric complex with the MUC1-N subunit at the cell membrane (1). To determine whether dimerization of MUC1-C is dependent on MUC1-N, we stably transfected human HCT116 colon cancer cells, which are null for MUC1 (20), to express full-length MUC1 (MUC1-N and MUC1-C) or only the MUC1-C subunit. As found in the breast and lung cancer cells, MUC1-C monomers and dimers were detectable in HCT116 cells expressing full-length MUC1 (HCT116/MUC1) (Fig. 2A). Immunoblot analysis of HCT116 cells expressing only MUC1-C (HCT116/MUC1-C) further demonstrated the presence of both MUC1-C momomers and dimers (Fig. 2B). Human LNCaP prostate cancer cells have undetectable levels of MUC1 expression (14). Consequently, to confirm the results in $\mathrm{HCT} 116$ cells, we transfected LNCaP cells to stably express MUC1-C (Fig. 2C). Immunoblot analysis of LNCaP/MUC1-C cells further demonstrated the detection of MUC1-C momomers and dimers (Fig. 2C). These findings indicate that MUC1-C forms homodimers by a mechanism independent of MUC1-N.

Dimerization of MUC1-C is detectable in the cytoplasm. Overexpression of MUC1, as found in carcinoma cells, is associated with accumulation of MUC1-C in the cytoplasm and thereby targeting of MUC1-C to the nucleus and mitochondrial outer membrane (1). To further assess the localization of MUC1-C dimers, we analyzed purified particulate and cytoplasmic fractions from ZR-75-1 cells. As a control, MUC1-C dimers were detectable in the whole cell lysate (Fig. 3A). Analysis of the particulate fraction demonstrated a relatively low level of dimers compared to that in the whole cell lysate (Fig. 3A). By contrast, MUC1-C dimers were readily detectable in the cytoplasmic fraction and comparable to that in the whole cell lysate (Fig. 3A). MUC1-C dimers were also detectable in the cytosolic fraction from $\mathrm{H} 1975$ cells (Fig. 3B). The cytoplasm is a reducing environment that prevents the formation of disulfide bond-mediated dimerization of many, but not all, proteins (19). 


\section{A MUC1-C Subunit}

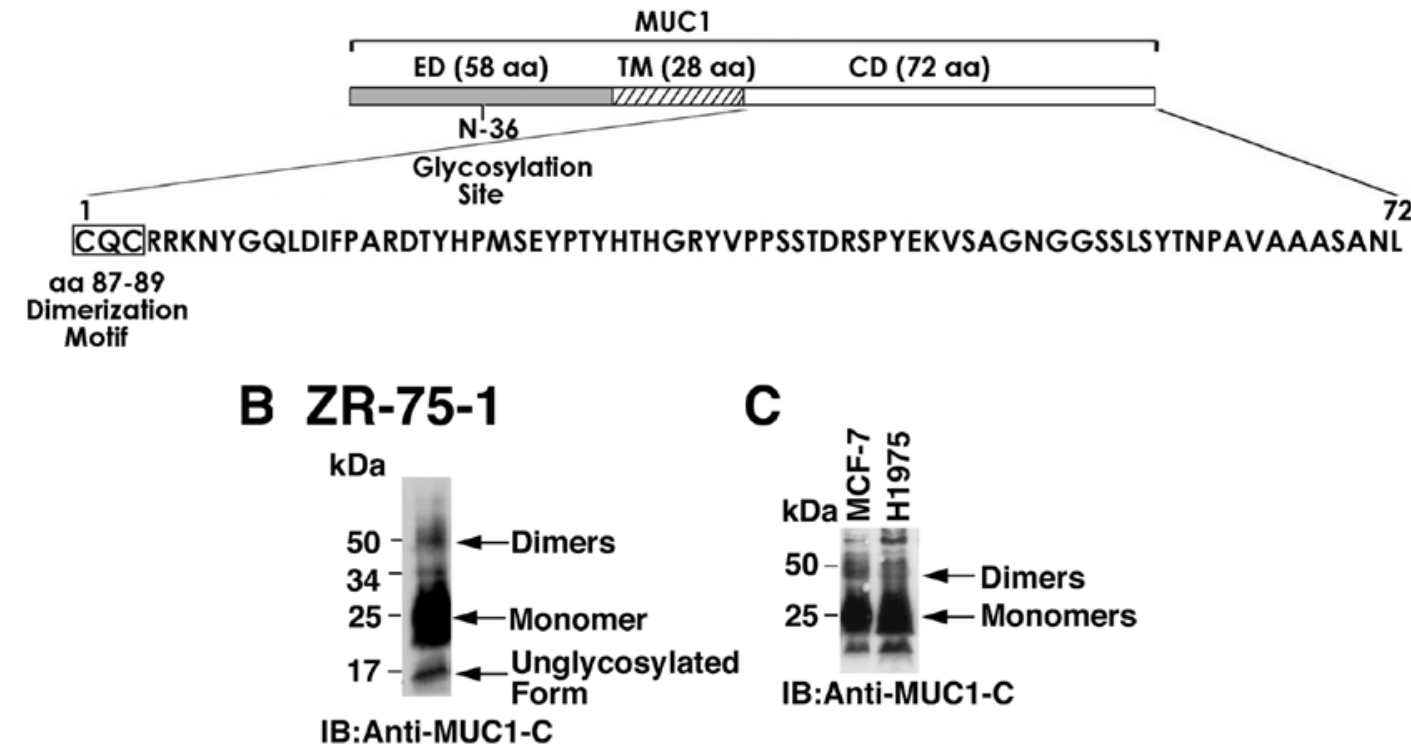

Figure 1. Dimerization of the MUC1-C subunit in cancer cells. (A) Schematic representation of the MUC1-C subunit and amino acid sequence of the cytoplasmic domain. Highlighted are the N-36 glycosylation site and the CQC motif at aa 87-89. (B and C) Total cell lysates from the indicated cells were separated in non-reducing SDS-polyacrylamide gels and immunoblotted with anti-MUC1-C.

\section{A HCT116/MUC1 B HCT116/MUC1-C C LNCaP/MUC1-C
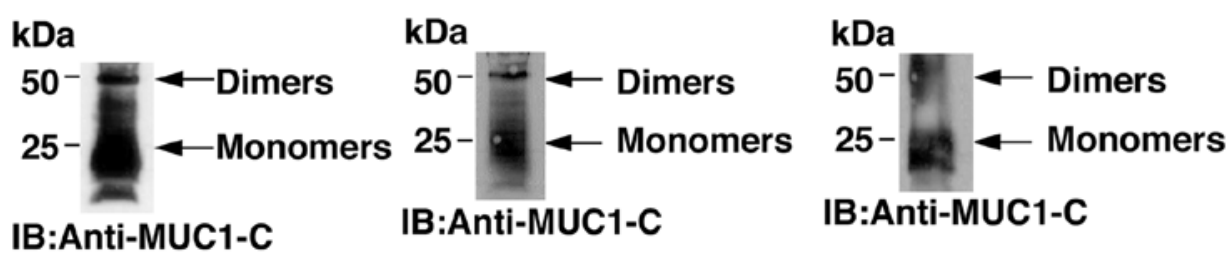

Figure 2. MUC1-C dimerization is detectable in the absence of MUC1-N. (A-C) Total cell lysates from HCT116/MUC1 (A), HCT116/MUC1-C (B) and LNCaP/ MUC1-C (C) cells were separated in non-reducing gels and immunoblotted with anti-MUC1-C.

\section{A ZR-75-1}

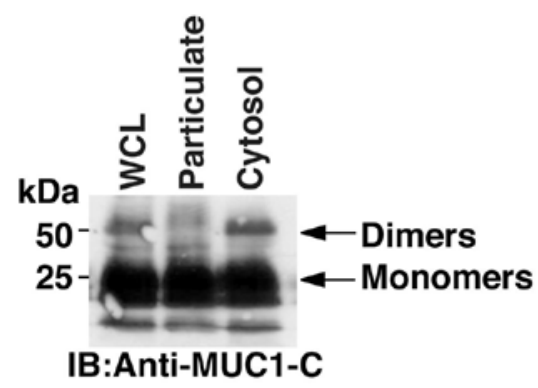

C ZR-75-1/Cytosol

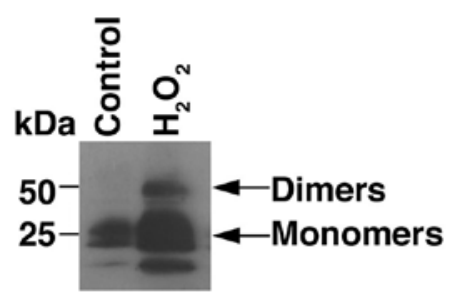

IB:Anti-MUC1-C

$43-$

IB:Anti- $\beta$-actin
B H1975/Cytosol

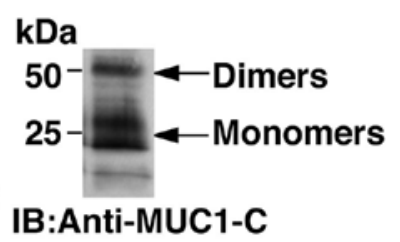

Figure 3. MUC1-C dimerization is detectable in the cytoplasm and increased by oxidative stress. (A) Whole cell lysate (WCL) and particulate and cytosolic lysates from ZR-75-1 cells were separated in non-reducing gels and immunoblotted with anti-MUC1-C. (B) Cytosolic lysate from H1975 cells was separated in non-reducing gels and immunoblotted with anti-MUC1-C. (C) ZR-75-1 cells were left untreated (control) and treated with $200 \mu \mathrm{M} \mathrm{H}_{2} \mathrm{O}_{2}$ for $1 \mathrm{~h}$. Cytosolic lysates were separated in non-reducing gels and immunoblotted with anti-MUC1-C and anti- $\beta$-actin. 
A

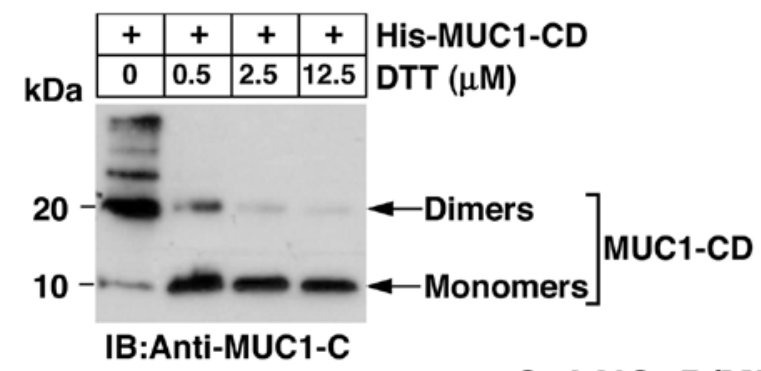

B

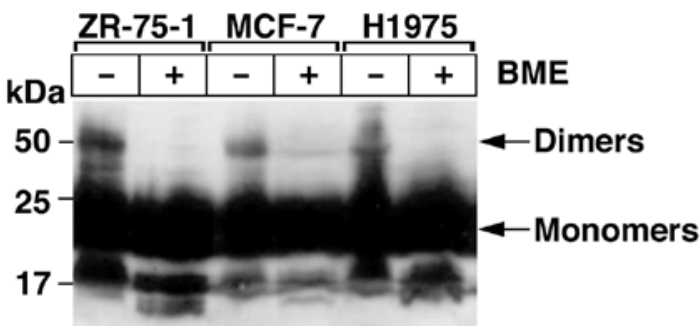

C LNCaP/MUC1-C

IB:Anti-MUC1-C

43

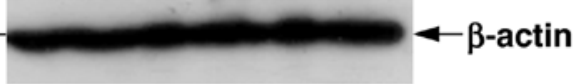

IB:Anti- $\beta$-actin

Figure 4. Disruption of MUC1-C dimerization by reducing agents. (A) Purified His-MUC1-CD was incubated with the indicated concentrations of DTT and then subjected to immunoblotting with anti-MUC1-C. (B and C) Lysates from the indicated cells were incubated in the absence and presence of $6 \%(0.8 \mathrm{mM})$ $\mathrm{BME}$, separated in non-reducing gels and then analyzed by immunoblotting with anti-MUC1-C.

To explore whether MUC1-C forms dimers in the cytoplasm through a thiol-dependent mechanism, we treated ZR-75-1 cells with hydrogen peroxide $\left(\mathrm{H}_{2} \mathrm{O}_{2}\right)$ to induce oxidative stress. Significantly, $\mathrm{H}_{2} \mathrm{O}_{2}$ treatment was associated with the upregulation of MUC1-C expression and increases in cytosolic MUC1-C dimers (Fig. 3C), indicating that MUC1-C forms dimers in the cytoplasm in the response to oxidative stress.

MUC1-C dimerization is disrupted by reducing agents. Immunoblot analyses of cell lysates as shown above demonstrate that MUC1-C dimerization is detectable when the analysis is performed in the absence of reducing agents. Therefore, to further assess the involvement of cysteine residues, we analyzed dimerization of the $\sim 10 \mathrm{kDa}$ His-tagged MUC1-C cytoplasmic domain (His-MUC1-CD) that contains the CQC motif. His-MUC1-CD forms homodimers and higher order oligomers that are detectable in the absence of a reducing agent (Fig. 4A). However, incubation of His-MUC1-CD in the presence of the reducing agent DTT resulted in a block of homodimerization (Fig. 4A). These results indicate that the reducing conditions are of importance for the detection of MUC1-C dimers. In this respect, immunoblot analysis of ZR-75-1 cell lysates demonstrated that MUC1-C dimers are disrupted by treatment with the reducing agent $\beta$-mercaptoethanol (BME) (Fig. 4B). Similar results were obtained when lysates from MCF-7 and H1975 cells were reduced with BME (Fig. 4B). In addition, analysis of LNCaP/MUC1-C cells demonstrated that MUC1-C dimers formed in the absence of MUC1-N are disrupted by BME (Fig. 4C). These findings indicate that MUC1-C homodimers are the result of disulfide bond formation through the CQC motif in the cytoplasmic domain.
MUC1-C dimerization is dependent on the CQC motif. To confirm that the MUC1-C CQC motif is necessary for dimerization, we studied HCT116 cells stably expressing MUC1 (HCT116/ MUC1) or MUC1 in which CQC was mutated to AQA [HCT116/ MUC1(AQA)] (12). The demonstration that MUC1-C dimers are detectable in HCT116/MUC1, but not in HCT116/MUC1(AQA), cells confirmed that MUC1-C dimerization is dependent on the CQC motif (Fig. 5A). Other studies of MUC1-C dimerization have employed MUC1-C fused with CFP and YFP proteins (21). In addition, these studies used the chemical cross-linker DSS to detect MUC1-C dimer formation. To first assess the effects of using a model in which MUC1-C is fused to GFP, we expressed GFP in human 293 kidney cells and asked if GFP itself forms dimers. In this context, GFP contains two Cys residues that play a role in its oligomerization (22). Indeed, our results demonstrate that expression of GFP in cells is associated with the formation of homodimers as detected in the presence of DSS cross-linking (Fig. 5B). Similar results were obtained with the CFP and YFP proteins (Fig. 5B). These findings demonstrate that the MUC1-C dimerization requires the $\mathrm{CQC}$ motif cysteines and that studies using MUC1-C fused with GFP, CFP or YFP can lead to experimental artifacts due to the propensity of these tags to form dimers.

Targeting the MUC1-C CQC motif disupts MUC1-C dimerization in cells. The demonstration that the MUC1-C CQC motif is of potential importance to MUC1-C function (12) prompted studies on how to block these cysteine residues. Accordingly, we synthesized cell-penetrating peptides that include the CQCRRKN sequence, such that the peptide binds directly to the MUC1-C cytoplasmic domain and thereby blocks its avail- 
A.

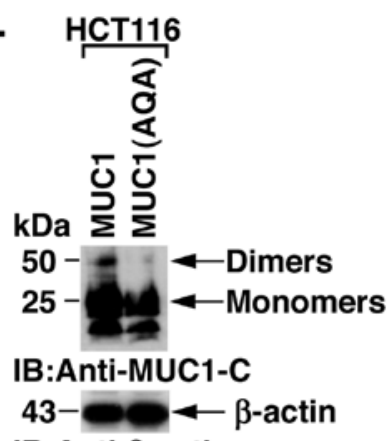

B.

IB:Anti- $\beta$-actin

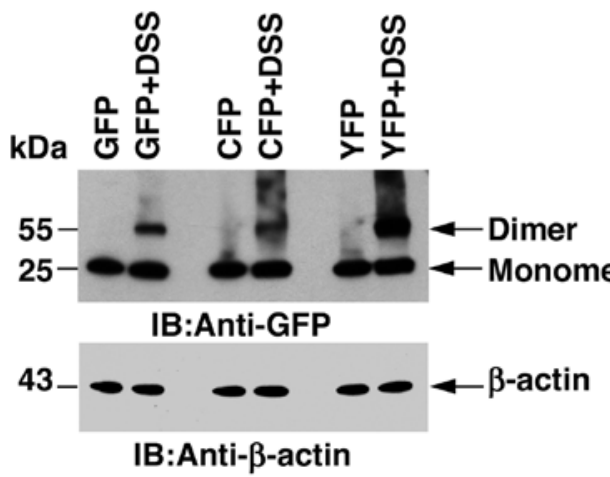

Figure 5. The MUC1-C CQC motif is necessary for dimerization. (A) Lysates from HCT116/MUC1 and HCT116/MUC1(AQA) cells were separated in nonreducing gels and analyzed by immunoblotting with the indicated antibodies. (B) Cells (293) were transfected to express GFP, CFP or YFP. Lysates were incubated in the absence and presence of DSS and analyzed by immunoblotting with the indicated antibodies. ability to form a dimer with another MUC1-C molecule (9). Studies with the CQCRRKN-containing peptides demonstrated that His-MUC1-CD dimerization in vitro is blocked using this approach (9). By contrast and as a control, AQARRKNcontaining peptides that do not bind to the MUC1-C cytoplasmic domain had no effect on His-MUC1-CD dimerization (9). To assess the effects of these peptides on MUC1-C dimerization in cells, we used GO-203, which contains a poly-Arg cell-penetrating domain linked to CQCRRKN (16) (Fig. 6A). Treatment of ZR-75-1 cells with $5 \mu \mathrm{M}$ GO-203 blocked MUC1-C dimerization (Fig. 6B). GO-203 also blocked MUC1-C dimerization in $\mathrm{H} 1975$ cells (Fig. 6C). In concert with these results, GO-203 was effective in blocking the formation of MUC1-C dimers in the cytoplasm (Fig. 6D). These findings demonstrate that targeting the MUC1-C CQC motif blocks the MUC1-C dimerization in cancer cells.

\section{Discussion}

Detection of MUC1-C subunit dimerization. The MUC1-C oncoprotein contributes to the malignant phenotype by interacting with receptor tyrosine kinases (RTKs) at the cell membrane, by regulating gene transcription in the nucleus and by blocking stress-induced cell death at the mitochondrial outer membrane (1). The available evidence indicates that MUC1-C dimerization is necessary for forming complexes with RTKs and for intracellular trafficking to the nucleus and mitochondria $(1,12,20)$. How MUC1-C forms homodimers is thus of importance for developing strategies to block its oncogenic function. The

\section{A. GO-203 [R $]_{9}-$ CQCRRKN D-amino acids}

B.

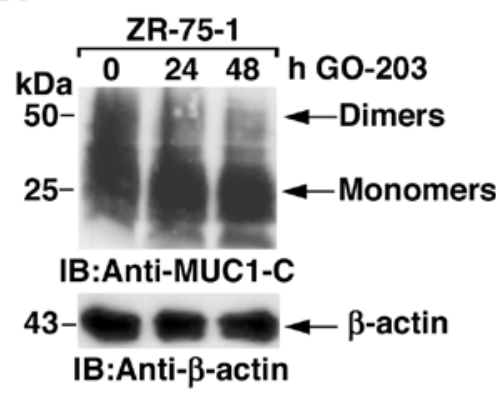

C.

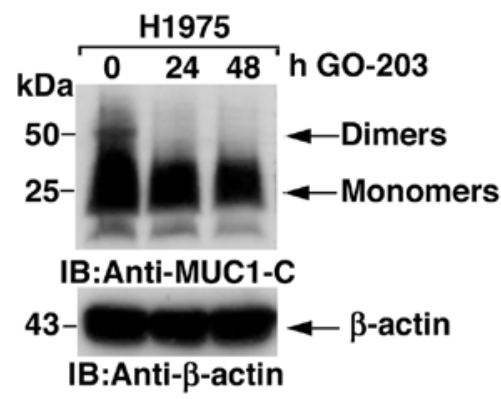

D. ZR-75-1/Cytosol

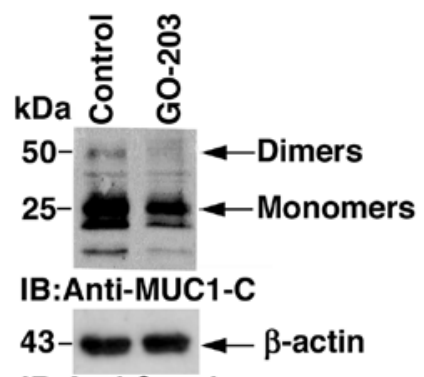

IB:Anti- $\beta$-actin

Figure 6. Targeting the MUC1-C CQC motif blocks dimerization. (A) D-amino acid sequence of GO-203 with the poly-Arg cell transduction domain and the CQCRRKN sequence that binds to the MUC1-C CQC motif. (B and C) ZR-75-1 (B) and H1975 (C) cells were treated with $5 \mu$ M GO-203 each day for two days. Whole cell lysates were separated in non-reducing gels and analyzed by immunoblotting with the indicated antibodies. (D) ZR-75-1 cells were treated with $5 \mu \mathrm{M}$ GO-203 each day for two days. Cytosolic lysates were separated in non-reducing gels and analyzed by immunoblotting with the indicated antibodies. 
MUC1-C cytoplasmic domain contains two cysteine residues that contribute to the formation of dimers in vitro (12). However, further studies were needed to assess the role of these cysteines in MUC1-C dimerization in cancer cells. Indeed, recent studies suggested that MUC1-C homodimers are formed by an unclear mechanism that does not involve cysteine residues (21). The interpretation of those results is complicated by the analysis of MUC1 fusion proteins containing CFP or YFP tags, and by using DSS cross-linking (21). As has been shown for GFP (22), expression of fusion proteins with CFP or YFP tags can result in misleading results as a result of their own dimerization. Accordingly, in the present work, expression of CFP and YFP in 293 cells demonstrated that these proteins clearly self-associate when the analysis is performed using DSS as a cross-linker (Fig. 5B). Based on these results, dimerization studies of MUC1-C-CFP or MUC1C-YFP fusion proteins in cells are confounded by the presence of these tags that self-associate and can be detected with DSS. The same argument applies to other tags such as CD8. Therefore, to avoid such misleading findings, analysis of MUC1-C dimerization in the present work has been performed with the subunit itself that is devoid of other self-associating proteins. Under these circumstances, our results show that MUC1-C forms higher order structures consistent with homodimers. The results also demonstrate that mutation of the cysteine residues abrogates the formation of MUC1-C dimers in cells. These findings indicate that the MUC1-C CQC motif cysteines are necessary and sufficient for MUC1-C homodimerization.

Dimerization of MUC1-C in the cytoplasm. The present results further demonstrate that MUC1-C forms dimers in the cytoplasm of carcinoma cells. These findings are in concert with previous observations that MUC1-C dimerization is necessary for targeting of cytoplasmic MUC1-C to the nucleus (12). Notably, the cytoplasm contains a high concentration of reduced glutathione that counteracts the formation of disulfide bonds (23). However, certain cytosolic proteins contain cysteine residues with low pKa values that function as thiol-based redox switches in response to reactive oxygen species (ROS) (19). For example, cytosolic glyceraldehyde-3-phosphate dehydrogenase (GAPDH) has an active site cysteine that is subject to oxidation, including the formation of intramolecular disulfide bonds (24). As a result, GAPDH enzymatic activity is inhibited with the attenuation of glycolysis (19). ROS-induced active site thiol modifications that include formation of disulfide bonds also occur in protein tyrosine phosphatases (PTPs; for example, PTP1B and PTEN) and thereby regulate their activity (19). Indeed, disulfide bond formation in diverse cytosolic proteins serves as a redox switch to modulate their functions $(19,25)$. Cytoplasmic MUC1-C appears to be one of these thiol-based redox signaling proteins. Indeed, the present results demonstrate that cytoplasmic MUC1-C is regulated by ROS, such that oxidation of the CQC motif cysteines in the response to hydrogen peroxide treatment increases the formation of MUC1-C dimers. Notably, redox balance is disrupted in diverse cancer cells as a result of increased ROS generation, indicating that treatment with agents that contribute to oxidative stress could be an effective therapeutic approach $(26,27)$. In this context, inhibition of MUC1-C dimerization with cell-penetrating peptide inhibitors is associated with increases in ROS $(13-16,28)$. Thus, MUC1-C inhibitors would in turn increase their propensity to form disul- fide bonds with the MUC1-C cytoplasmic domain and further promote redox imbalance and cell death. Consistent with these findings, overexpression of MUC1-C as found in carcinoma cells blocks stress-induced increases in ROS, further supporting a role for the MUC1-C cytoplasmic domain cysteines as sensors of the cellular redox state (29-32).

Targeting MUC1-C dimerization as a potential cancer treatment. Human HCT116 colon cancer cells are null for MUC1-C expression and thus represent a model for studying the effects of MUC1-C mutants (20). In this way, HCT116 cells were transfected to stably express wild-type MUC1 or MUC1 in which the two cysteines in the MUC1-C cytoplasmic domain were mutated to alanines [MUCl(AQA)] (12). Wild-type MUC1 increased HCT116 colony formation and stimulated tumor cell growth in nude mice (12). Interestingly, HCT116 cells expressing MUC1(AQA) exhibited a marked decrease in tumorigenicity, indicating that this mutant may function as a dominant-negative for transformation (12). To extend these findings, cell-penetrating peptides were synthesized to block the MUC1-C CQC motif. In concert with the importance of the CQC motif cysteines in MUC1-C dimerization as noted above, the present results further demonstrate that GO-203 inhibits the detection of MUC1-C dimers in breast and lung cancer cells. Importantly, treatment of breast and lung cancer cells with GO-203 or the first generation MUC1-C inhibitory peptides (GO-201,GO-202) inhibited growth and induced death in vitro $(13,16)$. These MUC1-C dimerization inhibitors also induced regressions of established breast and lung tumor xenografts growing in nude mice $(13,16)$. Those findings and the present results collectively support a model in which blocking MUC1-C homodimerization could be an effective strategy for cancer treatment. In that line of reasoning, the firstin-man peptide MUC1-C inhibitor has entered Phase I evaluation in patients with refractory solid tumors.

\section{Acknowledgements}

This work was supported by Grants CA97098, CA42802 and CA100707 awarded by the National Cancer Institute. D.K. holds equity in Genus Oncology and is a consultant to the company. D.R., G.P. and S.K. are employees of Genus Oncology. The other authors disclose no potential conflicts of interest.

\section{References}

1. Kufe D: Mucins in cancer: function, prognosis and therapy. Nat Rev Cancer 9: 874-885, 2009.

2. Kufe D, Inghirami G, Abe M, Hayes D, Justi-Wheeler H and Schlom J: Differential reactivity of a novel monoclonal antibody (DF3) with human malignant versus benign breast tumors. Hybridoma 3: 223-232, 1984

3. Ramasamy S, Duraisamy S, Barbashov S, Kawano T, Kharbanda S and Kufe D: The MUC1 and galectin-3 oncoproteins function in a microRNA-dependent regulatory loop. Mol Cell 27: 992-1004, 2007.

4. Li Y, Ren J, Yu W, et al: The EGF receptor regulates interaction of the human DF3/MUC1 carcinoma antigen with c-src and ß-catenin. J Biol Chem 276: 35239-35242, 2001.

5. Li Y, Liu D, Chen D, Kharbanda S and Kufe D: Human DF3/ MUC1 carcinoma-associated protein functions as an oncogene. Oncogene 22: 6107-6110, 2003.

6. Huang L, Chen D, Liu D, Yin L, Kharbanda S and Kufe D: MUC1 oncoprotein blocks GSK3 $\beta$-mediated phosphorylation and degradation of $\beta$-catenin. Cancer Res 65: 10413-10422, 2005 . 
7. Yamamoto M, Bharti A, Li Y and Kufe D: Interaction of the DF3/MUC1 breast carcinoma-associated antigen and $\beta$-catenin in cell adhesion. J Biol Chem 272: 12492-12494, 1997.

8. Wei X, Xu H and Kufe D: Human MUC1 oncoprotein regulates p53-responsive gene transcription in the genotoxic stress response. Cancer Cell 7: 167-178, 2005.

9. Ahmad R, Raina D, Joshi MD, Kawano T, Kharbanda S and Kufe D: MUC1-C oncoprotein functions as a direct activator of the NF-kB p65 transcription factor. Cancer Res 69: 7013-7021, 2009.

10. Ahmad R, Rajabi H, Kosugi M, et al: MUC1-C oncoprotein promotes STAT3 activation in an auto-inductive regulatory loop. Sci Signal 4: ra9, 2011.

11. Kufe D: Oncogenic function of the MUC1 receptor subunit in gene regulation. Oncogene 29: 5663-5666, 2010.

12. Leng Y, Cao C, Ren J, et al: Nuclear import of the MUC1-C oncoprotein is mediated by nucleoporin Nup62. J Biol Chem 282: 19321-19330, 2007.

13. Raina D, Ahmad R, Joshi M, et al: Direct targeting of the MUC1 oncoprotein blocks survival and tumorigenicity of human breast carcinoma cells. Cancer Res 69: 5133-5141, 2009.

14. Joshi MD, Ahmad R, Raina D, et al: MUC1 oncoprotein is a druggable target in human prostate cancer cells. Mol Cancer Ther 8: 3056-3065, 2009.

15. Yin L, Ahmad R, Kosugi M, et al: Survival of human multiple myeloma cells is dependent on MUC1 c-terminal transmembrane subunit oncoprotein function. Mol Pharm 78: 166-174, 2010.

16. Raina D, Kosugi M, Ahmad R, et al: Dependence on the MUC1-C oncoprotein in non-small cell lung cancer cells. Mol Cancer Ther 10: 806-816, 2011.

17. Panchamoorthy G, Rehan H, Kharbanda A, Ahmad R and Kufe D A monoclonal antibody against the oncogenic mucin 1 cytoplasmic domain. Hybridoma (In press).

18. Rhee SG, Bae YS, Lee SR and Kwon J: Hydrogen peroxide: a key messenger that modulates protein phosphorylation through cysteine oxidation. Sci STKE 2000: pe1, 2000

19. Brandes N, Schmitt S and Jakob U: Thiol-based redox switches in eukaryotic proteins. Antioxid Redox Signal 11: 997-1014, 2009.

20. Ren J, Agata N, Chen D, et al: Human MUC1 carcinoma-associated protein confers resistance to genotoxic anti-cancer agents. Cancer Cell 5: 163-175, 2004.
21. Bernier AJ, Zhang J, Lillehoj E, Shaw AR, Gunasekara N and Hugh JC: Non-cysteine linked MUC1 cytoplasmic dimers are required for src recruitment and ICAM-1 binding induced cell invasion. Mol Cancer 10: 93, 2011.

22. Jain RK, Joyce PB, Molinete M, Halban PA and Gorr SU: Oligomerization of green fluorescent protein in the secretory pathway of endocrine cells. Biochem J 360: 645-649, 2001.

23. Ostergaard $\mathrm{H}$, Tachibana $\mathrm{C}$ and Winther JR: Monitoring disulfide bond formation in the eukaryotic cytosol. J Cell Biol 166: 337-345, 2004.

24. Leichert LI, Gehrke F, Gudiseva HV, et al: Quantifying changes in the thiol redox proteome upon oxidative stress in vivo. Proc Natl Acad Sci USA 105: 8197-8202, 2008.

25. Linke K and Jakob U: Not every disulfide lasts forever: disulfide bond formation as a redox switch. Antioxid Redox Signal 5: 425-434, 2003

26. Trachootham D, Alexandre J and Huang P: Targeting cancer cells by ROS-mediated mechanisms: a radical therapeutic approach? Nat Rev Drug Discov 8: 579-591, 2009.

27. Ogasawara MA and Zhang H: Redox regulation and its emerging roles in stem cells and stem-like cancer cells. Antioxid Redox Signal 11: 1107-1122, 2009.

28. Yin L, Wu Z, Avigan D, et al: MUC1-C oncoprotein suppresses reactive oxygen species-induced terminal differentiation of acute myelogenous leukemia cells. Blood 117: 4863-4870, 2011.

29. Yin L and Kufe D: Human MUC1 carcinoma antigen regulates intracellular oxidant levels and the apoptotic response to oxidative stress. J Biol Chem 278: 35458-35464, 2003.

30. Yin L, Huang L and Kufe D: MUC1 oncoprotein activates the foxo3a transcription factor in a survival response to oxidative stress. J Biol Chem 279: 45721-45727, 2004.

31. Yin L, Kharbanda S and Kufe D: Mucin 1 oncoprotein blocks hypoxia-inducible factor 1 alpha activation in a survival response to hypoxia. J Biol Chem 282: 257-266, 2007.

32. Yin L, Kharbanda S and Kufe D: MUC1 oncoprotein promotes autophagy in a survival response to glucose deprivation. Int J Oncol 34: 1691-1699, 2009. 